ARIADNE FERREIRA AMARAL ${ }^{1}$

EDUARDo Silva Araújo²

JULIANa CRISTINA MaGalhães ${ }^{2}$

ÉRIKa Aparecida SILVERA ${ }^{1}$

Suelene Brito do Nascimento Tavares ${ }^{3}$

RItA GoReti AMARAL ${ }^{2}$

Artigo Original

Palavras-chave

Educação continuada Capacitação em serviço Capacitação profissional Capacitação de recursos humanos em saúde Neoplasias do colo do útero/diagnóstico Neoplasias do colo do útero/prevenção \& controle

Keywords

Education, continuing Inservice training

Professional training

Health human resource training Uterine cervical neoplasms/diagnosis Uterine cervical neoplasms/prevention \& control

\section{Impacto da capacitação dos profissionais de saúde sobre o rastreamento do câncer do colo do útero em unidades básicas de saúde}

\author{
Impact of training about cervical cancer screening on bealth \\ professionals working in basic health care units
}

\section{Resumo}

OBJETIVO: Avaliar o impacto da capacitação dos profissionais envolvidos no rastreamento do câncer do colo do útero (CCU) em Unidades Básicas de Saúde (UBSs) do município de Goiânia (GO). MÉTODOS: Estudo de intervenção cujas variáveis estudadas foram: os dados pessoais da mulher, anamnese, exame clínico e identificação do profissional responsável pela coleta (informações contidas no formulário de requisição do exame citopatológico do colo do útero). A avaliação da capacitação dos profissionais foi realizada por meio da comparação dos formulários referentes ao período de janeiro de 2007 a abril de 2009, antes da capacitação, com os formulários de julho de 2010 a dezembro de 2012 , após a capacitação. Para avaliar o resultado da capacitação foi utilizado o teste do $\chi^{2}$ de Pearson, com nível de significância de 5\%. RESULTADOS: Após a capacitação, houve aumento significativo da frequência de preenchimento da escolaridade, de 67,2 para $92,6 \%(p<0,001)$, do telefone, de 78,9 para $98,7 \%$ ( $p<0,001)$, da inspeção do colo, de 86,8 para 96,6\% (p<0,001) e sinais sugestivos de doenças sexualmente transmissíveis (DST), de 80,8 para $93,5 \%$ (p<0,001). Houve redução da frequência de realização do exame no intervalo menor que um ano $(p<0,001)$ e de um ano $(p<0,001)$. Houve redução da frequência de realização do exame citopatológico em mulheres com menos de 25 anos de idade, de 22,0 para 17,9\% ( $p<0,001)$. Houve um aumento significativo da proporção de amostras satisfatórias, de 70,4 para 80,2\% (p<0,001). Foi verificada redução da ocorrência de fatores obscurecedores. A frequência de dessecamento foi de 2,9\% antes da capacitação e de 2,0\% após a capacitação $(p<0,001)$. Houve um aumento da frequência de representação de células endocervicais, de 79,5 para 88,5\% (p<0,001). CONCLUSÃO: Após a capacitação, houve melhora expressiva no preenchimento do formulário de requisição, na realização do exame citopatológico, conforme a periodicidade e a faixa etária preconizadas pelo Ministério da Saúde, e na adequabilidade da amostra.

\section{Abstract}

PURPOSE: To evaluate the impact of training professionals involved in the screening for cervical cancer in Basic Health Units in the city of Goiânia (GO). METHODS: This was and intervention study in which the following data contained in the cervical cytopathology test form were examined: the woman's personal data, anamnesis, clinical examination and identification of the professional responsible for the collection. Professional training was evaluated by comparing the forms referring to the period from January 2007 to April 2009, before training, with the forms referring to the period from July 2010 to December 2012, after training. The Pearson $\chi^{2}$ test was used to analyze the results of training, with the $5 \%$ level of significance. RESULTS: After training, there was a significantly increased frequency of recording patient schooling lfrom 67.2 to $92.6 \%, p<0.001$ ), telephone number (from 78.9 to $98.7 \%, p<0.001$ ), cervical inspection (from 86.8 to $96.6 \%, p<0.001$ ), and signs suggestive of sexually transmitted diseases (from 80.8 to 93.5\%, $p<0.001$ ). There was a reduction in the frequency of performing the exam within an interval of less than one year $(p<0.001)$ and of one year $(p<0.001)$. There was a reduction in the frequency of Pap smear testing in
Correspondência

Rita Goreti Amaral

Foculdade de Farmácio Universidade Federal de Goís $1^{0}$ Avenida, esquina com a Proca Universitánía, $\mathrm{s} / \mathrm{n}$ - Setor Leste

Universitário (EP. 74645-220 Goiânia (G0), Brasil

Recebido $06 / 02 / 2014$
Centro de Análises Clínicas Rômulo Rocha da Faculdade de Farmácia da Universidade Federal de Goiás - UFG - Goiânia (GO), Brasil.

'Programa de Pós-Graduação em Ciências da Saúde, Universidade Federal de Goiás - UFG - Goiânia (GO), Brasil.

2Faculdade de Farmácia, Universidade Federal de Goiás - UFG - Goiânia (GO), Brasil.

3Departamento de Citologia, Faculdade de Farmácia, Universidade Federal de Goiás - UFG - Goiânia (GO), Brasil.

Conflito de interesses: não há 
women under 25 years of age, from 22.0 to $17.9 \%$ ( $p<0.001)$. There was a significant increase in the proportion of satisfactory samples from 70.4 to $80.2 \%(p<0.001)$. A reduction of confounding factors was observed. The desiccation frequency was $2.9 \%$ before training and $2.0 \%$ after training $(p<0.001)$. There was an increase in the frequency of representation of endocervical cells from 79.5 to $88.5 \%(p<0.001)$. CONCLUSION: After training, there was a significant improvement in completing the application form, the performance of such tests regarding frequency and the age range recommended by the Ministry of Health, and the adequacy of the sample.

\section{Introdução}

O câncer do colo do útero (CCU) é considerado um grave problema de saúde pública no mundoํำ Os países em desenvolvimento são os principais responsáveis pelas altas taxas de mortalidade 2 . A alta incidência de tal neoplasia é resultado da exposição das mulheres aos fatores de risco e da falta de efetividade de um programa de rastreamento ${ }^{3}$.

A redução da incidência e mortalidade do CCU só é possível por intermédio da detecção das lesões precursoras em mulheres assintomáticas, por meio do exame citopatológico do colo do útero, fundamental para a eficiência de um programa de rastreamento organizado ${ }^{4}$. É necessário seguir um conjunto de ações programadas, com a população-alvo, faixa etária e a periodicidade entre os exames bem definidas 5 .

O Ministério da Saúde, em $2010^{6}$, elaborou propostas para o aperfeiçoamento técnico e operacional do programa nacional de controle do CCU. Essas propostas foram sistematizadas em cinco eixos. O eixo um é caracterizado pelo fortalecimento do rastreamento organizado na atenção primária e da gestão descentralizada do programa de rastreamento ${ }^{6}$. Neste eixo, recomenda-se fortalecer a formação e qualificação dos profissionais de saúde envolvidos nas ações do rastreamento organizado, ampliar a divulgação das recomendações e condutas para o controle do CCU e aprimorar o Sistema de Informação do Controle do Câncer do Colo do Útero (SISCOLO) ${ }^{6}$.

Para garantir o rastreamento organizado é necessário o preenchimento correto do formulário de requisição do exame citopatológico do colo do útero ${ }^{7}$. A falha no preenchimento pode impedir o cadastramento da mulher no sistema, a busca ativa daquelas com resultados alterados, gerar erros no diagnóstico, dificuldades na entrega do resultado ou até mesmo falta de dados necessários para a realização do exame ${ }^{7}$.

Outro ponto fundamental para o sucesso do rastreamento é a adequabilidade da amostra ${ }^{8}$. A qualidade das amostras cervicais pode ser avaliada pela presença de células da região ectocervical, do canal endocervical e da junção escamo-colunar, onde se localiza a maioria dos carcinomas cervicais e lesões precursoras ${ }^{9}$.

Devido aos erros que podem ocorrer durante a fase pré-analítica, é necessário que os profissionais de saúde ${ }^{8}$ tenham uma educação permanente, visto que a coleta inadequada levará a erros de diagnóstico, elevando a ocorrência de resultados falsos-negativos ${ }^{8}$.

A prática do rastreamento do CCU, na maioria das vezes, não segue as recomendações do Ministério da Saúde a periodicidade mais adotada pelos profissionais é a anual, e têm sido rastreadas mulheres fora da faixa etária recomendada, que até 2011 era de 25 a 59 anos de idade e, a partir deste ano o recomendado passou a ser 25 a 64 anos de idade ${ }^{10}$. Além disso, muitas mulheres com resultados citopatológicos alterados não são orientadas e nem encaminhadas adequadamente de acordo com as condutas clínicas preconizadas ${ }^{5,11,12}$. Estas diferenças entre a prática vigente e as normas do Ministério da Saúde podem ser um fator de dificuldade para o êxito do rastreamento organizado ${ }^{13}$.

Diante da necessidade de melhorar a qualidade do exame citopatológico para o rastreamento do CCU, o objetivo deste estudo foi avaliar o impacto da capacitação dos profissionais de saúde envolvidos no rastreamento do CCU em Unidades Básicas de Saúde (UBSs).

\section{Métodos}

Este foi um estudo de intervenção realizado pela Faculdade de Farmácia da Universidade Federal de Goiás (FF-UFG) em parceria com a Secretaria Municipal de Saúde de Goiânia e aprovado pelo Comitê de Ética da Universidade, sob o protocolo $\mathrm{n}^{\circ}$ 022/2012.

A casuística foi constituída a partir das informações contidas nos formulários de requisição dos exames citopatológicos do colo do útero das mulheres atendidas em sete UBSs de Goiânia (GO): Parque Amendoeiras, Recanto das Minas Gerais, Vila Pedroso, Santo Hilário, Jardim Don Fernando II, Deputado João Natal e Setor Leste Universitário.

A escolha dessas UBSs ocorreu devido ao desenvolvimento de estudos anteriores e parceria da FF-UFG nessas regiões do município de Goiânia (GO) no desenvolvimento de atividades de ensino, pesquisa e extensão.

Os formulários foram preenchidos nas UBSs pelos médicos e enfermeiros responsáveis pela coleta da amostra. Os exames citopatológicos foram analisados por especialistas no setor de citologia clínica do Centro de Análises Clínicas Rômulo Rocha da FF-UFG.

Foram avaliados 10.672 formulários referentes ao período de janeiro de 2007 a abril de 2009, antes da capacitação, e 10.672, de julho de 2010 a dezembro de 
2012, após a capacitação, totalizando 21.344 formulários. Os formulários de requisição referentes ao período de capacitação que ocorreu entre maio de 2009 e junho de 2010 foram excluídos da análise. A capacitação foi realizada pelos pesquisadores da FF-UFG e pelos profissionais da Secretaria Municipal de Saúde.

A metodologia empregada na capacitação foi fundamentada na aprendizagem significativa e educação dialógica, na qual o professor atua como facilitador do processo de ensino-aprendizagem, estimulando o aprendiz a ter postura ativa, crítica e reflexiva durante a construção do conhecimento ${ }^{14,15}$.

O treinamento dos profissionais envolvidos no rastreamento do CCU foi realizado em duas etapas. $\mathrm{Na}$ primeira etapa foram capacitados os médicos, enfermeiros e técnicos de enfermagem; na segunda etapa, os agentes comunitários de saúde.

Para o treinamento dos médicos, enfermeiros e técnicos de enfermagem foram abordados os seguintes temas: como funciona o Programa Nacional de Prevenção do Câncer do Colo do Útero no Brasil, a importância do SISCOLO, do preenchimento adequado do formulário de requisição do exame citopatológico, coleta adequada do exame, a faixa etária e a periodicidade recomendadas, conduta clínica e seguimento das mulheres com resultado do exame alterado.

Para o treinamento dos agentes comunitários de saúde foram abordados os seguintes temas sobre o CCU: fatores de risco, prevenção, quem deve fazer, de quanto em quanto tempo, sinais de alerta e como deve ser realizada a busca ativa das mulheres que nunca realizaram o exame, aquelas com intervalo maior que três anos e com resultados citopatológicos alterados.

A avaliação do impacto da capacitação dos profissionais envolvidos no rastreamento do CCU foi realizada por meio da comparação das informações contidas nos formulários de requisição dos exames citopatológicos realizados antes e após a capacitação.

As variáveis utilizadas na pesquisa foram: os dados pessoais da mulher, anamnese, exame clínico, identificação do profissional, periodicidade, faixa etária, adequabilidade da amostra e os resultados dos exames citopatológicos ${ }^{9,16}$

O banco de dados foi estruturado no Epi Info ${ }^{\circledR}$ versão 3.5.1. O programa Stata ${ }^{\circledR}$ versão 12.0 foi utilizado para o processamento e análise dos dados ${ }^{17}$. Para verificar a distribuição das variáveis, foi utilizado o cálculo de frequências absolutas e relativas e para avaliação do impacto da capacitação foi utilizado o teste do $\chi^{2}$ de Pearson, com nível de significância de $5 \%$.

\section{Resultados}

Foram analisados 21.344 formulários, que contemplaram os períodos antes e após a capacitação. A média de idade

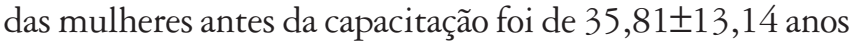
e, após a capacitação, foi de 37,94士13,74 anos.

Houve aumento significativo da frequência de preenchimento da escolaridade, de 67,2 para $92,6 \%$ $(\mathrm{p}<0,001)$, após a capacitação. Houve aumento significativo da frequência de preenchimento do telefone, de 78,9 para $98,7 \%(\mathrm{p}<0,001)$, após a capacitação. Houve aumento significativo da frequência de preenchimento da identidade, de 75,6 para 98,9\% ( $\mathrm{p}<0,001)$, após a capacitação. Também houve aumento significativo da frequência de preenchimento do endereço, de 99,8 para 99,9\% ( $\mathrm{p}<0,005)$, após a capacitação.

Houve aumento significativo da frequência de preenchimento da inspeção do colo, de 86,8 para 96,6\% ( $\mathrm{p}<0,001$ ), e sinais sugestivos de doenças sexualmente transmissíveis (DST), de 80,8 para $93,5 \%(\mathrm{p}<0,001)$ após a capacitação. Também houve aumento significativo da frequência de preenchimento da identificação do profissional, de 98,5 para 99,4\% ( $<<0,001$ ) (Tabela 1). Outros dados são apresentados na Tabela 1.

Tabela 1. Frequência de preenchimento das informações no formulário de requisição do exame citopatológico do colo do útero, antes e após a capacitação, em unidades básicas de saúde

\begin{tabular}{|c|c|c|c|c|c|}
\hline \multirow{2}{*}{ Variáveis } & \multicolumn{2}{|c|}{ Antes da capacifação } & \multicolumn{2}{|c|}{ Após a capacitação } & \multirow{2}{*}{ Valor $\mathrm{p}$} \\
\hline & n & $\%$ & n & $\%$ & \\
\hline Fez exame preventivo alguma vez & 9.773 & 91,6 & 10.166 & 95,3 & 0,01 \\
\hline Usa DIU & 10.342 & 96,8 & 10.381 & 97,3 & 0,1 \\
\hline Está grávida & 10.672 & 97,3 & 10.401 & 97,5 & 0,4 \\
\hline Usa pílula anticoncepcional & 10.312 & 96,6 & 10.328 & 96,8 & 0,5 \\
\hline Já fez tratamento por radioterapia & 7.672 & 71,9 & 9.056 & 84,9 & 0,01 \\
\hline Tem ou teve algum sangramento após relações sexuais & 9.407 & 88,2 & 9.511 & 89,4 & 0,01 \\
\hline Tem ou teve algum sangramento após menopausa & 7.268 & 68,1 & 8.862 & 83,0 & 0,01 \\
\hline Inspeção do colo & 9.268 & 86,8 & 10.315 & 96,6 & 0,01 \\
\hline Sinais sugestivos de DST & 8.621 & 80,8 & 9.974 & 93,5 & 0,01 \\
\hline
\end{tabular}

Teste do $\chi^{2}(p<0,05)$.

DST: doenças sexualmente transmissíveis. 
Considerando-se a periodicidade do exame citopatológico, antes e após a capacitação, houve redução da frequência de realização do exame no intervalo menor que um ano $(5,3$ versus $2,8 \% ; \mathrm{p}<0,001)$ e de um ano $(40,1$ versus $31,4 \%$; $\mathrm{p}<0,001)$, respectivamente. Houve um aumento da frequência de realização do exame nos intervalos 2 anos (25,3 versus $29,9 \% ; \mathrm{p}<0,001), 3$ anos $(10,4$ versus $15,7 \% ; \mathrm{p}<0,001)$ e maior que 3 anos $(10,2$ versus $11,6 \% ; \mathrm{p}<0,005)$. Houve redução da frequência de realização do exame citopatológico em mulheres com menos de 25 anos de idade, de 22,0 para $17,9 \%(p<0,001)$, após a capacitação. Houve aumento da frequência de realização do exame citopatológico em mulheres de 25 a 59 anos de idade, de 72,4 para 74,6\% (p<0,001), e em mulheres com mais de 59 anos de idade, de 5,6 para $7,4 \%(\mathrm{p}<0,001)$, após a capacitação. Houve um aumento significativo da proporção de amostras satisfatórias, de 70,4 para 80,2\% (p<0,001), após a capacitação (Tabela 2).

Foi verificada redução da ocorrência de fatores obscurecedores. A frequência de dessecamento foi de 2,9\% antes da capacitação e de 2,0\% após a capacitação $(\mathrm{p}<0,001)$. Houve um aumento da frequência de representação de células endocervicais, de 79,5 para 88,5\% ( $\mathrm{p}<0,001)$, após a capacitação. Outros dados são apresentados na Tabela 3.

Tabela 2. Variação da adequabilidade da amostra e do resultado do exame citopatológico do colo do útero, antes e após a capacitação, em unidades básicas de saúde

\begin{tabular}{|c|c|c|c|c|c|}
\hline & \multicolumn{2}{|c|}{$\begin{array}{c}\text { Antes da } \\
\text { capacitaç̃ão }\end{array}$} & \multicolumn{2}{|c|}{$\begin{array}{c}\text { Após a } \\
\text { capacitação }\end{array}$} & \multirow[t]{2}{*}{ Valor $\mathrm{p}$} \\
\hline & n & $\%$ & n & $\%$ & \\
\hline \multicolumn{6}{|l|}{ Adequabilidade da amostra } \\
\hline Satisfatória & 7.404 & 70,4 & 8.425 & 80,2 & 0,01 \\
\hline Satisfatória com fator obscurecedor & 2.836 & 27,0 & 1.803 & 17,2 & 0,01 \\
\hline Insatisfatória & 271 & 2,6 & 281 & 2,7 & 0,7 \\
\hline \multicolumn{6}{|l|}{ Resultado do exame citopatológico } \\
\hline Negativo & 9.486 & 92,6 & 9.445 & 92,3 & 0,4 \\
\hline Lesões menos graves & 510 & 5,0 & 506 & 4,9 & 0,9 \\
\hline Lesões mais graves & 244 & 2,4 & 277 & 2,7 & 0,1 \\
\hline
\end{tabular}

Para esta análise, foram excluídas as amostras insatisfatórias; teste do $\chi^{2}(p<0,05)$.

Tabela 3. Adequabilidade da amostra satisfatória com fator obscurecedor e representação do epitélio para células endocervicais e metaplásicas, antes e após capacitação, em unidades básicas de saúde

\begin{tabular}{|c|c|c|c|c|c|}
\hline & \multicolumn{2}{|c|}{$\begin{array}{c}\text { Antes da } \\
\text { capacitação }\end{array}$} & \multicolumn{2}{|c|}{$\begin{array}{c}\text { Após a } \\
\text { capacitação }\end{array}$} & \multirow[t]{2}{*}{ Valor $p$} \\
\hline & n & $\%$ & n & $\%$ & \\
\hline \multicolumn{6}{|c|}{ Satisfatória com fator obscurecedor } \\
\hline Dessecamento & 308 & 2,9 & 217 & 2,0 & 0,01 \\
\hline Purulento & 483 & 4,5 & 373 & 3,5 & 0,01 \\
\hline Sangue & 95 & 0,9 & 52 & 0,5 & 0,01 \\
\hline Áreas espessas & 93 & 0,9 & 65 & 0,6 & 0,01 \\
\hline Outras causas & 81 & 0,8 & 45 & 0,4 & 0,01 \\
\hline \multicolumn{6}{|l|}{ Representação do epitélio } \\
\hline Células endocervicais & 8.485 & 79,5 & 9.445 & 88,5 & 0,01 \\
\hline Células metaplásicas & 9.400 & 88,1 & 9.505 & 89,1 & 0,01 \\
\hline
\end{tabular}

Teste do $\chi^{2}(p<0,05)$.

\section{Discussão}

Os resultados deste estudo mostraram que, após a capacitação dos profissionais da saúde, houve uma melhora significativa no preenchimento do formulário de requisição dos exames citopatológicos do colo do útero referente aos dados pessoais da mulher, da anamnese e do exame clínico.

Sabe-se que o preenchimento do formulário de requisição do exame citopatológico do colo do útero é um dos elementos da fase pré-analítica fundamentais para a qualidade deste exame, que consequentemente refletirá na qualidade do programa de rastreamento ${ }^{7}$. A falta de informações para a realização do exame citopatológico pode impedir o cadastramento da mulher no sistema e a identificação das mulheres que fazem parte do grupo de risco para o desenvolvimento de lesões precursoras do $\mathrm{CCU}^{10}$.

O preenchimento correto dessas informações é de extrema importância, pois auxilia o profissional a interpretar os resultados citopatológicos durante a análise dos exames no laboratório, além de possibilitar a identificação dos casos que devem ser revisados de acordo com o recomendado pelo controle interno de qualidade do Ministério da Saúde 7 .

Nesta pesquisa também foi possível verificar um aumento significativo da identificação do profissional responsável pela coleta, o que, consequentemente, diminuiu a devolução dos exames às UBSs ${ }^{7}$. A devolução do formulário de requisição devido à falta de preenchimento correto pode provocar atraso na entrega do resultado para a mulher ${ }^{9}$. Além de causar transtornos na rotina do serviço, gera custos adicionais ao sistema de saúde ${ }^{11}$.

Neste estudo não foi observado aumento do número de exames das mulheres que informaram realizá-los pela primeira vez, provavelmente devido às deficiências relacionadas a problemas na organização da rede de atenção à saúde primária ${ }^{5}$. O que justifica o fato de que, no Brasil, o rastreamento ainda é oportunístico e a maioria das mulheres procura os serviços de saúde por razões adversas à prevenção do $\mathrm{CCU}^{6,7}$.

Os principais motivos que influenciaram algumas mulheres a nunca ter realizado o exame citopatológico foram: o desconhecimento sobre o CCU e sobre a importância do exame, medo de doer e de se deparar com resultado positivo para câncer, sentimento de vergonha e dificuldades em realizar o exame, como falta de dinheiro para locomoção, acesso ao serviço de saúde e ao emprego ${ }^{18}$.

A partir da realização deste estudo foi observado que a frequência dos exames citopatológicos realizados nos intervalos menor que um ano e um ano foi significativamente menor após a capacitação. No intervalo de três anos houve um aumento significativo dessa frequência. 
O Ministério da Saúde preconiza que mulheres que iniciaram o rastreamento, tiveram exames com amostras insatisfatórias e apresentaram alterações devem repetir o exame em intervalo menor ou igual a um ano ${ }^{9}$. Aquelas que realizaram o exame citopatológico por dois anos consecutivos e apresentaram resultados negativos devem realizar o exame a cada três anos ${ }^{6}$.

Para os intervalos de dois anos e maior que três anos houve aumento significativo da frequência de exames, fator que pode ser explicado tendo em vista a resistência das mulheres e dos profissionais de saúde em seguir as recomendações do Ministério da Saúde.

Em países que adotaram o rastreamento organizado, com a realização do exame citopatológico a cada 3 anos, as taxas de mortalidade por CCU caíram cerca de $40 \%$. A incidência diminuiu mais de um terço na Austrália e na Nova Zelândia e cerca de um quinto na Inglaterra ${ }^{19}$.

Em relação à frequência dos exames citopatológicos realizados em mulheres com menos de 25 anos de idade, houve uma redução após a capacitação. O Ministério da Saúde recomenda que o rastreamento do CCU deve ter início aos 25 anos de idade ${ }^{6,9}$, visto que a incidência do CCU em mulheres com até 24 anos é muito baixa. A maioria dos casos é diagnosticada como lesões de baixo grau, consideradas não precursoras e que possuem grande probabilidade de regressão ${ }^{7}$.

Recomendações semelhantes têm sido adotadas por outros países. Os Estados Unidos da América indicavam o rastreio após o início da atividade sexual; a partir de 2002, o país passou a adotar o prazo de 3 anos, com limite máximo aos $21 \operatorname{anos}^{20}$. Na Europa, é recomendado que o rastreamento inicie entre 25 e 30 anos de idade ${ }^{21}$.

A frequência dos exames citopatológicos realizados na faixa etária de 25 a 59 anos e maior que 59 anos aumentou significativamente. A faixa etária de 25 a 59 anos é considerada a de maior risco para o desenvolvimento das lesões precursoras e do CCU, sendo prioridade do Ministério da Saúde principalmente as mulheres que nunca realizaram o exame ${ }^{9}$. O aumento da frequência dos exames realizados em mulheres com mais de 59 anos pode ser justificado na ação do Ministério da Saúde, que, em 2011, publicou as Diretrizes Brasileiras para o Rastreamento do Câncer do Colo do Útero, preconizando que a população-alvo a ser rastreada deve pertencer à faixa etária de 25 aos 64 anos de idade ${ }^{10}$.

Sasieni et al..$^{22}$ mostraram que o rastreamento em mulheres com idade entre 20 e 24 anos teve pequeno ou nenhum impacto sobre as taxas de câncer cervical invasivo. Em contraste, o rastreamento em mulheres idosas obteve uma substancial redução na incidência e mortalidade em decorrência do CCU. Mulheres em torno de 40 anos de idade apresentaram uma redução de $60 \%$ dos casos de câncer, aumentando para $80 \%$ na faixa etária de 64 anos, parcela na qual o rastreamento foi particularmente eficaz na prevenção do câncer em estágio invasivo.

Foi possível verificar, neste estudo, que as amostras insatisfatórias não apresentaram diferença significativa após a capacitação, mas a frequência foi menor que $3 \%$. Segundo o Instituto Nacional do Câncer, em 2005, apenas seis Estados brasileiros e o Distrito Federal apresentaram taxas de exames insatisfatórios menores que $5 \%$, padrão mínimo de qualidade estabelecido pela Organização PanAmericana de Saúde 2 . Por outro lado, foi observado, após a capacitação, um aumento significativo da frequência das amostras satisfatórias e uma redução das amostras com fatores obscurecedores, indicando uma melhora da qualidade da coleta do exame citopatológico do colo do útero.

A coleta adequada do material é muito importante para o êxito do diagnóstico citopatológico. A garantia da presença do material em quantidades suficientes é fundamental para o sucesso da ação $0^{7,9}$.

Foi observada, em relação às amostras satisfatórias com algum fator obscurecedor, uma diminuição significativa para todas as causas (dessecamento, purulento, áreas espessas e sangue). Houve, ainda, um aumento da representação das células endocervicais e/ou metaplásicas após a capacitação, o que pode ter ocorrido devido ao melhor entendimento dos profissionais sobre a importância de se realizar uma coleta adequada com representação da junção escamo-colunar.

Esperava-se encontrar diferença significativa para o resultado do exame citopatológico, uma vez que houve redução das amostras satisfatórias com fator obscurecedor e melhor representação das células endocervicais e metaplásicas. Ainda que não tenha sido observada uma maior quantidade significativa de lesões detectadas após a capacitação, para as lesões mais graves houve um discreto aumento.

Tal resultado pode ser atribuído ao fato de não ter aumentado a frequência de exames realizados pela primeira vez, podendo inferir que as mulheres que realizaram os exames citopatológicos eram as mesmas, consequentemente, não apresentou um aumento na detecção das lesões precursoras.

O estudo de Amaral et al. ${ }^{8}$ mostrou que os principais fatores que limitaram a análise dos exames citopatológicos foram a ausência de células endocervicais, dessecamento, purulento e áreas espessas ${ }^{8}$. Dias et al. ${ }^{23}$ mostraram que os principais fatores que prejudicaram a análise dos exames citopatológicos foram dessecamento, presença de sangue e material escasso, enfatizado pelos estudos que isso decorre de problemas na coleta e fixação inadequadas da lâmina.

Diante dos resultados obtidos neste estudo, é possível concluir que, após a capacitação dos profissionais envolvidos na realização do exame citopatológico do colo do útero, houve melhora expressiva no preenchimento do formulário 
de requisição, na realização do exame, conforme a periodicidade e a faixa etária preconizadas pelo Ministério da Saúde, e na adequabilidade da amostra.

Estes resultados servirão de subsídios para a implementação de capacitações dos profissionais envolvidos no rastreamento do CCU, com o intuito de melhorar a qualidade do exame citopatológico e, consequentemente, reestruturar o programa de rastreamento oportunístico para um programa organizado. Ficou evidente o quanto é importante qualificar e atualizar os profissionais da atenção básica à saúde sobre as normas e condutas adotadas com relação à realização do exame citopatológico para o rastreamento do CCU.

\section{Agradecimentos}

Ao Professor Dr. Délio Marques Conde, por suas importantes considerações sobre este estudo, à equipe do laboratório Rômulo Rocha da Faculdade de Farmácia da Universidade Federal de Goiás e à Coordenação de Aperfeiçoamento de Pessoal de Nível Superior (CAPES), pelo apoio financeiro.

\section{Referências}

1. Katz LM, Souza AS, Fittipaldi SO, Santos GM, Amorim MM. [Agreement between cytology, colposcopy and cervical histopathology]. Rev Bras Ginecol Obstet. 2010;32(8):368-73. Portuguese.

2. Thuler LC. [Mortality due to cancer of the uterine cervix in Brazil]. Rev Bras Ginecol Obstet. 2008;30(5):216-8. Portuguese.

3. Corrêa DA, Villela WV. O controle do câncer do colo do útero: desafios para implementação de ações programáticas no Amazonas, Brasil. Rev Bras Saúde Mater Infant. 2008;8(4):491-7.

4. Löwy I. Cancer, women, and public health: the history of screening for cervical cancer. Hist Ciênc Saúde-Manguinhos. 2010; 17(Suppl 1):53-67.

5. Vale DB, Morais SS, Pimenta AL, Zeferino LC. Avaliação do rastreamento do câncer do colo do útero na Estratégia Saúde da Família no Município de Amparo, São Paulo, Brasil. Cad Saúde Pública. 2010;26(2):383-90.

6. Brasil. Ministério da Saúde. Instituto Nacional de Câncer. Plano de ação para redução da incidência e mortalidade por câncer do colo do útero: sumário executivo. Rio de Janeiro: INCA; 2010.

7. Brasil. Ministério da Saúde. Secretaria de Atenção à Saúde. Departamento de Atenção Básica. Controle dos cânceres do colo do útero e da mama. 2a ed. Brasília: Ministério da Saúde; 2013. (Cadernos de Atenção Básica; 13).

8. Amaral RG, Manrique EJ, Guimarães JV, Sousa PJ, Mignoli JR, Xavier AF, et al. [Influence of adequacy of the sample on detection of the precursor lesions of the cervical cancer]. Rev Bras Ginecol Obstet. 2008;30(1 1):556-60. Portuguese.

9. Brasil. Ministério da Saúde. Secretaria de Assistência à Saúde. Instituto Nacional de Câncer. Coordenação de Prevenção e Vigilância. Nomenclatura brasileira para laudos cervicais e condutas preconizadas: recomendações para profissionais de saúde. 2a ed. Rio de Janeiro: INCA; 2006.

10. Brasil. Ministério da Saúde. Instituto Nacional de Câncer. Coordenação Geral de Ações Estratégicas. Divisão de Apoio à Rede de Atenção Oncológica. Diretrizes brasileiras para o rastreamento do câncer do colo do útero. Rio de Janeiro: INCA; 2011

11. Martins LF, Valente JG, Thuler LC. Factors related to inadequate cervical cancer screening in two Brazilian state capitals. Rev Saúde Pública. 2009;43(2):318-25.
12. Freitas RA, Carvasan GA, Morais SS, Zeferino LC. Excessive Pap smears due to opportunistic cervical cancer screening. Eur J Gynaecol Oncol. 2008;29(5):479-82.

13. Albuquerque ZB, Manrique EJ, Tavares SB, Silva e Souza AC, Guimarães JV, Amaral RG. [Women with atypical, precursor lesions and invasive cervical cancer: behaviors according to the recommendations of the Ministry of Health]. Rev Bras Ginecol Obstet. 2012;34(6):248-53. Portuguese.

14. Alves VS. [A health education model for the Family Health Program: towards comprehensive health care and model reorientation]. Interface (Botucatu). 2005;9(16):39-52. Portuguese.

15. Chiesa AA, Veríssimo ML. A educação em saúde na prática do PSF. In: Brasil. Instituto para o Desenvolvimento da Saúde. Universidade de São Paulo. Ministério da Saúde. Manual de enfermagem. Brasília: Ministério da Saúde; 2001 . p. 34-42.

16. Solomon D, Nayar R. Sistema Bethesda para citopatologia cervicovaginal. 2a ed. Rio de Janeiro: Revinter; 2005.

17. StataCorp. Stata: data analysis and statistical software: release 12. College Station: StataCorp; 2011.

18. Ferreira ML. Motivos que influenciam a não-realização do exame de Papanicolaou segundo a percepção de mulheres. Esc Anna Nery. 2009; 13(2):378-84.

19. Simonella L, Canfell K. The impact of a two- versus three-yearly cervical screening interval recommendation on cervical cancer incidence and mortality: an analysis of trends in Australia, New Zealand, and England. Cancer Causes Control. 2013;24(9):1727-36.

20. Smith RA, Cokkinides V, Brooks D, Saslow D, Shah M, Brawley OW. Cancer screening in the United States, 2011: a review of current American Cancer Society guidelines and issues in cancer screening. CA Cancer J Clin. 2011;61(1):8-30.

21. Arbyn M, Antilla A, Jordan J, Ronco G, Schenck U, Segnan N, et al. European Guidelines for Quality Assurance in Cervical Cancer Screening. Second edition summary document. Ann Oncol. 2010;21(3):448-58.

22. Sasieni P, Castanon A, Cuzick J, Snow J. Effectiveness of cervical screening with age: population based case-control study of prospectively recorded data. BM. 2009;339:b2968.

23. Dias MB, Tomazelli JG, Assis M. Rastreamento do câncer de colo do útero no Brasil: análise de dados do Siscolo no período de 2002 a 2006. Epidemiol Serv Saúde. 2010;19(3):293-306. 